\title{
SELECTIVIDAD Y PRECARIEDAD LABORAL EN LA MIGRACIÓN CALIFICADA DE AMÉRICA LATINA Y EL CARIBE, 2000-2010
}

\author{
Selectivity and precarious labor in the highly skilled migration from \\ Latin America and the Caribbean, 2000-2010
}

\author{
Telésforo Ramírez García \\ Fernando Lozano Ascencio ${ }^{2}$
}

\begin{abstract}
Resumen. El objetivo central de este trabajo es examinar los principales cambios en los niveles y tendencias de la migración calificada global entre 2000 y 2010, con especial énfasis en lo que acontece en América Latina y el Caribe (ALyC). El artículo toma como ejes analíticos la existencia de políticas inmigratorias selectivas de este tipo de mano de obra en los países de destino, una limitada incorporación en las economías de origen, así como una inserción laboral precaria de los migrantes calificados en las economías del Norte global. La información que se emplea es la base de datos recientemente publicada por la Organización para la Cooperación y Desarrollo Económicos (OCDE) conocida como DIOC 2000/01 y 2010/11. El artículo da cuenta de un incremento acelerado de la migración calificada en ALyC (particularmente para el caso de las mujeres), un aumento de la "fuga de cerebros" entre 2000 y 2010 y una inserción laboral crecientemente precaria y desfavorable para los migrantes calificados de ALyC.
\end{abstract}

Palabras clave: migración calificada, fuga de cerebros, América Latina y el Caribe, selectividad migratoria, sobrecalificación.

\begin{abstract}
The aim of this paper is to examine the main changes in the levels and trends of global highly skilled migration between 2000 and 2010, with special emphasis on what happens in the Latin America and the Caribbean region (LAC). The paper takes as analytical axes the selective immigration policies of highly skilled migrants in the countries of destination, the limited incorporation of them in the origin countries, and their precarious labor incorporation in the economies of the global North. The information used here is the recently published database by the Organization for Economic Co-operation and Development (OECD) known as DIOC 2000/01 and 2010/11. The article shows an accelerated increase in skilled labor migration (particularly in the case of women), an increase in the "brain drain" between

1 Catedrático Conacyt en el Centro Regional de Investigaciones Multidisciplinarias (CRIM) de la

2 Centro Regional de Investigaciones Multidisciplinarias (CRIM) de la UNAM. Cuernavaca,
\end{abstract} UNAM. Cuernavaca, Morelos, México. Morelos, México. 
2000 and 2010, and an increasingly precarious and unfavorable labor insertion for highly skilled migrants of LAC.

Keywords: highly skilled migration, brain drain, Latin American and the Caribbean, migration selectivity and over-qualification.

\section{Introducción}

Una de las expresiones más sobresalientes de la migración internacional en el arranque del siglo XXI ha sido el importante crecimiento de la migración altamente calificada (grupo conformado por personas con educación terciaria), a un ritmo incluso más acelerado que la migración de media y baja calificación. Este comportamiento, que se ha observado en el orden global, tuvo un significativo repunte en los países que conforman la región de América Latina y el Caribe (ALyC). ¿Cómo explicar este acelerado crecimiento de la migración calificada en la región? ¿Se trata de un crecimiento atribuible a la situación económica y social de los países de destino o a los de origen? ¿En qué condiciones se está llevando a cabo la incorporación de los migrantes calificados en las sociedades de destino?

Factores de diversa índole están detrás de este crecimiento de la migración calificada. Uno primero tiene que ver con las políticas inmigratorias impulsadas en los países del Norte global para atraer mano de obra calificada $y$, de esa manera, cubrir las necesidades de trabajadores especializados de su aparato productivo. Se trata de políticas muy selectivas orientadas a reclutar a los "mejores y más brillantes", es decir, a trabajadores con determinadas habilidades, formados en áreas del conocimiento y/o niveles de especialización específicos. Como ejemplo de estas políticas -que algunos autores denominan como "quality-selectivity" immigration policies- están las que se impulsan en países como Australia, Canadá o Estados Unidos³. Simplemente en este último país, en 2013 estaban vigentes 911 mil visas de trabajadores especializados, bajo el esquema de visas $\mathrm{H}-1 \mathrm{~B}, \mathrm{~L}-1$ y $\mathrm{F}-1^{4}$.

Otros factores a considerar en la explicación de este incremento de la migración calificada son los vinculados con lo que sucede en los países de origen de los migrantes. Así vemos que durante la primera década del presente siglo, se presentó un incremento de la llamada "fuga de cerebros" en muchos países de América Latina y el Caribe, pues pese a que entre los años 2000 y 2010 la región latinoamericana tuvo incrementos también históricos en la formación de recursos humanos con nivel terciario, esta población de profesionistas calificados no se incorporó en el mercado laboral de sus respectivos países de

\footnotetext{
3 ANTECOL, Heather, COBB-CLARK, Deborah, TREJO, Stephen. Immigration policy and the skills of immigrants to Australia, Canada and the United States.

${ }^{4}$ DEPARTMENT OF HOMELAND SECURITY. Yearbook of Immigration Statistics, 2015.
} 
origen con esa misma intensidad con que creció y, por el contrario, incrementó sus niveles de emigración tanto en términos absolutos como relativos, tal y como lo veremos más adelante. A esta situación se suma el hecho que históricamente en estos países ha prevalecido la migración de personas que poseen una escolaridad mayor, proceso calificado por diversos autores -sobre todo por aquellos que estudian a la migración desde una perspectiva económica- como selectividad migratoria positiva, ya que existe una autoselección de acuerdo con ciertas características socioeconómicas como sexo, edad, escolaridad, entre otras ${ }^{5}$. No obstante, en el análisis de ciertos circuitos migratorios, como el que prevalece entre México y Estados Unidos, existe una fuerte controversia en el sentido que no necesariamente predomina la emigración de personas con mayor escolaridad, sino lo opuesto, es decir un una selección negativa ${ }^{6}$.

Un tercer cuerpo de argumentos que es preciso tomar en cuenta para entender el significativo crecimiento de la migración calificada, tiene que ver con la calidad de la incorporación de esta población migrante en las sociedades de destino, específicamente la referida a su inserción laboral. Sobre este aspecto, en la literatura especializada ha sido ampliamente documentado el hecho de que la inserción de la población migrante, se da predominante en mercados laborales desregulados, en ocupaciones con bajos salarios, lo que significa una inserción laboral precaria, concepto que implica inestabilidad laboral, ausencia de esquemas de protección y limitado acceso a programas de seguridad social ${ }^{7}$. Este incremento de las desigualdades sociales y laborales, en relación con la población nativa y al interior de la propia población de migrantes calificados, se confirma por el hecho de que los trabajadores migrantes y más específicamente los de América Latina y el Caribe, ocupan los niveles más bajos de la estructura ocupacional aceptando trabajos con salarios mal remunerados o en ocupaciones que no se corresponden con su educación formal, situación que en este artículo se identifica como trabajo sobre-calificado o sobre-calificación laboral.

El objetivo central de este trabajo es examinar los principales cambios en los niveles y tendencias de la migración calificada global entre 2000 y 2010, con especial énfasis en lo que acontece en América Latina y el Caribe, tomando como ejes analíticos los argumentos enunciados, que podrían estar vinculados con el aumento de la migración calificada en ALyC. La fuente de información que se emplea es la base de datos sobre inmigrantes residentes en países de la Organización para la Cooperación y Desarrollo Económicos (OCDE) conocida

\footnotetext{
KANVUR, Ravin, RAPOPORT, Hillel. Migration selectivity and the evolution of spatial inequality. RENDALL, Michael, PARKER, Susan. Two Decades of Negative Educational Selectivity of Mexican Migrants to the United States.

ANDERSON, Bridget. Migration, immigration controls and the fashioning of precarious workers. RODGERS, Gerry, RODGERS, Janine. Precarious Jobs in Labour Market Regulation: The Growth of Atypical Employment in Western Europe.
} 
como DIOC 2000/01 y 2010/118. Se trata de una fuente de datos muy completa, ya que incluye información de la población inmigrante de 33 países de la OCDE, de quince años y más, por país de origen, sexo, nivel educativo, estatus ocupacional (empleo/desempleo), así como información sobre la sobrecalificación laboral de los migrantes. Pese a que se trata de una base de datos que incluye información de sólo 33 países de destino, se trata de los países más importantes en la inmigración de trabajadores calificados y no calificados.

La estructura del artículo es la siguiente. Después de esta introducción, se presenta un análisis de los niveles y tendencias de la migración calificada en el nivel global, seguido por una sección que examina las principales tendencias de esta migración en la región latinoamericana, por subregiones y países de origen, regiones de destino y tasas de emigración calificada. Continúa con un análisis de la inserción socioeconómica de los migrantes calificados de ALyC en los países de la OCDE, por niveles de ocupación y sobre-calificación, para concluir con algunas reflexiones finales.

\section{Grandes tendencias de la migración calificada en el mundo}

Hasta finales del siglo XX, las grandes corrientes migratorias en el mundo se constituían mayoritariamente por personas con bajos niveles de escolaridad, quienes emigraban masivamente de sus países de origen, en respuesta a la demanda de mano de obra de baja calificación en diversos sectores económicos de los países desarrollados. Sin embargo, en años recientes, se ha observado una cada vez mayor participación de personas con alta escolaridad (licenciatura terminada y/o posgrado) en los flujos migratorios internacionales. La evidencia empírica muestra que tras haber comenzado como casos aislados en los años cincuenta ${ }^{9}$, la migración calificada se incrementó en las siguientes décadas y se diversificó durante los noventa y primer decenio del actual milenio, dando como resultado una alta concentración de población migrante calificada en países como Canadá, Estados Unidos, España, Reino Unido, entre otros.

Al respecto, la información de las bases de datos DIOC 2000/01 y DIOC 2010/11, revelan que entre 2000 y 2010, la población de migrantes calificados en los países de la OCDE pasó de 17.8 millones en 2000 a 31.3 millones en 2010, lo que en términos relativos representó un incremento de $75 \%{ }^{10}$. Tal crecimiento supera con creces al registrado por los migrantes con medio y bajo nivel

8 Database on Immigrants in OECD and non-OECD Countries: DIOC < http://www.oecd.org/els/ mig/dioc.htm>.

9 ESTEBAN, Fernando. La movilidad de profesionales y estudiantes universitarios latinoamericanos y caribeños a países de la OCDE.

10 La clasificación de los niveles calificación considerada en las bases de datos de la OCDE están definidos a partir de la escolaridad de las personas migrantes: bajo (hasta 8 años de escolaridad), medio (de 9 a 12 años de escolaridad) y alto (13 o más años de escolaridad). 
calificación (48.2\% y $13.8 \%$, respectivamente). De hecho, al analizar los cambios en la distribución porcentual de la población según su escolaridad entre 2000 y 2010, se observa que los migrantes con alto nivel de calificación representaban en el primer año el $23.6 \%$ del stock total de migrantes, aumentando a $29.6 \%$ en 2010. En cambio, la participación porcentual de los migrantes de bajo nivel de calificación, pese a que en términos absolutos aumentó en 4.3 millones de personas, disminuyó de $40.7 \%$ a 33.2\% entre 2000 y 2010. De igual forma, aunque la población migrante con un nivel calificación medio también aumentó significativamente al pasar de 24.8 a 36.8 millones, en términos relativos sólo creció en dos puntos porcentuales: $32.9 \%$ a $34.9 \%$ (ver tabla 1). Estas cifras revelan la existencia de una selectividad positiva por la población migrante con altos niveles de educación, al menos en países de la OCDE, tal y como se ha documentado en otros estudios sobre el tema ${ }^{11}$.

\section{Tabla 1 - Población migrante residente en los países de la OCDE, por nivel de escolaridad: 2000 y 2010}

\begin{tabular}{c|c|c|c|c|c}
\hline \multirow{2}{*}{ Nivel de escolaridad } & \multicolumn{2}{|c|}{2000} & \multicolumn{2}{c|}{2010} & \multirow{2}{*}{ Cambio \% } \\
\cline { 2 - 5 } & Abs. & $\%$ & Abs. & $\%$ & 2000-2010 \\
\hline Total & $75,715.9$ & 100.0 & $105,722.8$ & 100.0 & 39.6 \\
\hline Bajo (0 a 8 años) & $30,826.5$ & 40.7 & $35,088.8$ & 33.2 & 13.8 \\
\hline Medio (9 a 12 años) & $24,889.7$ & 32.9 & $36,890.6$ & 34.9 & 48.2 \\
\hline Alto (13 años o más) & $17,853.7$ & 23.6 & $31,352.0$ & 29.7 & 75.6 \\
\hline N.E. & $2,146.0$ & 2.8 & $2,391.3$ & 2.3 & 11.4 \\
\hline Hombres & $37,049.0$ & 100.0 & $51,173.1$ & 100.0 & 38.1 \\
\hline Bajo (0 a 8 años) & $14,728.8$ & 39.8 & $16,775.7$ & 32.8 & 13.9 \\
\hline Medio (9 a 12 años) & $12,263.0$ & 33.1 & $18,166.9$ & 35.5 & 48.1 \\
\hline Alto (13 años o más) & $9,041.5$ & 24.4 & $15,024.5$ & 29.4 & 66.2 \\
\hline N.E. & $1,015.7$ & 2.7 & $1,206.0$ & 2.4 & 18.7 \\
\hline Mujeres & $38,666.8$ & 100.0 & $54,549.7$ & 100.0 & 41.1 \\
\hline Bajo (0 a 8 años) & $16,097.7$ & 41.6 & $18,313.1$ & 33.6 & 13.8 \\
\hline Medio (9 a 12 años) & $12,626.7$ & 32.7 & $18,723.7$ & 34.3 & 48.3 \\
\hline
\end{tabular}

Fuente: Elaboración de los autores en base en OCDE: DIOC 2000/01 (archivo A_1_T) y DIOC 2010/11 (archivo A).

11 ARSLAN, Cansin, DUMONT, Jean-Christophe, KONE Zovanga et alii. International migration to the OECD in the twenty-first century; ORRENIUS, Pia, ZAVODNY, Madeline. Self-selection among undocumented immigrants from Mexico; MCKENZIE, David, RAPOPORT, Hillel. Self-selection patterns in Mexico-U.S. migration: the role of migration networks; LOZANO ASCENCIO, Fernando, GANDINI, Luciana. Migrantes calificados de América Latina y el Caribe ¿Capacidades desaprovechadas? 
No obstante, cabe subrayar que dicha selectividad adquiere distintos matices según sexo y nivel educativo de los migrantes, pues aunque hombres y mujeres con alto nivel educativo incrementaron su volumen absoluto entre 2000 y 2010 , las mujeres lo hicieron en mayor medida que los hombres: de 8.8 millones a 16.3 millones, y los hombres de 9 a 15 millones, lo que significa un incremento de $85.3 \%$ para las mujeres y $66.2 \%$ para los hombres en dicho periodo.

Estas cifras globales, sin embargo, esconden tendencias y patrones migratorios distintos según país o región de procedencia de los migrantes calificados. Asia, África y América Latina y el Caribe son las regiones del mundo de donde proviene la mayor parte de los migrantes calificados residentes en los países de la OCDE. El stock de migrantes calificados originarios de Asia aumentó de 5.9 a 11 millones de personas entre 2000 y 2010, en tanto que el de los nativos de Europa lo hizo de 6.1 a 10.8 millones, y el de los procedentes de América Latina y el Caribe de 2.6 a 4.8 millones. Estos tres grupos de migrantes calificados crecieron a un ritmo superior al promedio mundial que fue de $75.6 \%$. No obstante cabe destacar que, en términos relativos, los nativos de África crecieron en $83.2 \%$, situándose como el segundo grupo poblacional con el mayor crecimiento porcentual durante el periodo, sólo por debajo de los asiáticos (84.1\%) (ver gráfico 1).

\section{Gráfico 1 - Población migrante calificada residente en los países de la OECD, por región de origen y cambio porcentual entre 2000 y 2010}

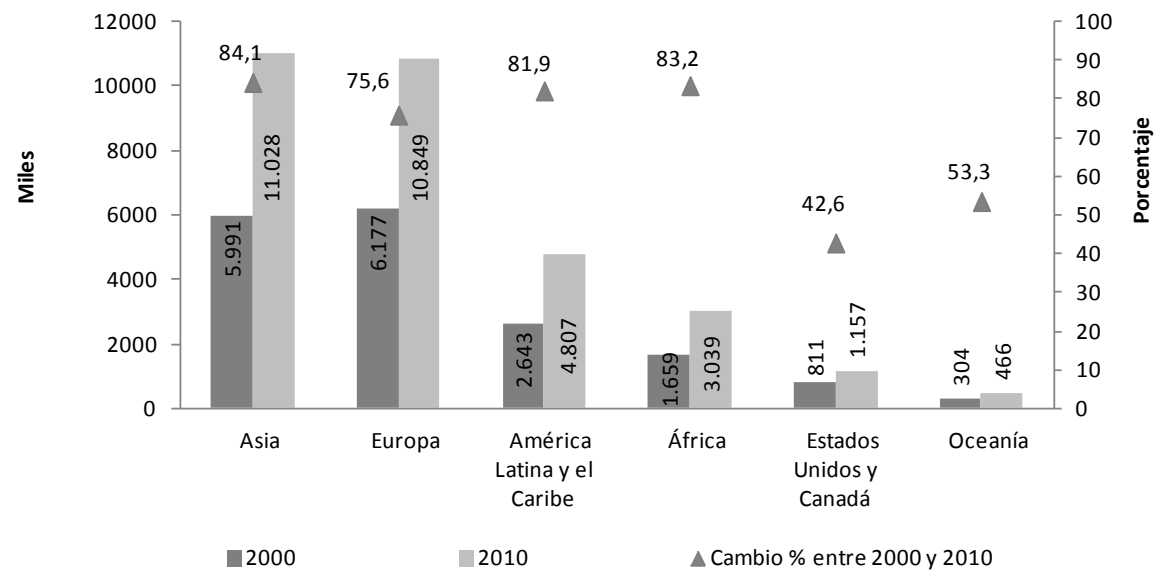

Fuente: Elaboración de los autores en base en OCDE: DIOC 2000/01 (archivo A_1_T) y DIOC 2010/11 (archivo A).

En términos generales, los datos hasta aquí presentados permiten confirmar y ratificar la persistencia de dos grandes tendencias de la migración calificada a nivel mundial: 1) el constante y sostenido crecimiento de la migración de recursos humanos calificados hacia países de la OCDE, con un ritmo superior al de la migración de personas con bajo o medio nivel de escolaridad; y 2) la creciente participación de las mujeres en el flujo migratorio de mano de obra 
calificada. Ambos procesos ya han sido enunciados en otras investigaciones sobre la temática ${ }^{12}$, en las que también se destacan cambios significativos en la magnitud y ritmo de crecimiento de la migración calificada entre regiones y subregiones de origen y destino.

\section{Migración calificada de latinoamericanos y caribeños hacia países de la OCDE}

Como se señaló líneas arriba, América Latina y el Caribe es una de las regiones del mundo con mayor migración calificada. Durante la última década (2000-2010), aportó alrededor del 15\% del total de los migrantes calificados residentes en los países de la OCDE. Asimismo, en ese mismo periodo, experimentó un crecimiento superior al promedio total (75.6\%), pasando de aportar 2.6 a 4.8 millones de personas, situándose por encima de otros países y regiones expulsoras de población calificada. De los países y subregiones que la conforman, la región Andina fue la que presentó el mayor crecimiento de la migración calificada en el periodo señalado (122.2\%), al pasar de 474 mil a poco más de un millón de migrantes. Dentro de esta subregión, destacan los casos de los nativos de Bolivia (179.6\%), Venezuela (145.1\%) y Perú (119.4\%). En segundo lugar se encuentra la región Sudamericana, la que en su conjunto creció en 109.5\%, aumentado su población migrante calificada de 332 a 696 mil personas, siendo Paraguay (239.1\%), Argentina (135.1\%) y Uruguay (112.7\%), los países que mayor número de migrantes calificados aportaron. En tercer lugar se encuentra México, país que registró un incremento de 86.8\%, pasando de 473 mil a 885 mil personas (ver tabla 2). Tabla 2 - Población migrante calificada residente en países de la OCDE,
por país y región de origen: 2000 y 2010

\begin{tabular}{l|c|c|c|c|c}
\hline \multirow{2}{*}{ Región de origen } & \multicolumn{2}{c|}{2000} & \multicolumn{2}{c}{2010} & $\begin{array}{c}\text { Cambio \% } \\
2000-2010\end{array}$ \\
\cline { 2 - 6 } & Abs. & $\%$ & Abs. & $\%$ & Total \\
\hline Total & 17.853 .676 & 100,0 & 31.352 .049 & 100,0 & 75,6 \\
\hline África & 1.658 .526 & 9,3 & 3.038 .961 & 9,7 & 83,2 \\
\hline Asia & 5.991 .363 & 33,6 & 11.027 .655 & 35,2 & 84,1 \\
\hline Europa & 6.176 .969 & 34,6 & 10.848 .642 & 34,6 & 75,6 \\
\hline Oceanía & 303.598 & 1,7 & 465.516 & 1,5 & 53,3 \\
\hline $\begin{array}{l}\text { Estados Unidos y } \\
\text { Canadá }\end{array}$ & 811.263 & 4,5 & 1.157 .121 & 3,7 & 42,6 \\
\hline $\begin{array}{l}\text { América Latina y el } \\
\text { Caribe }\end{array}$ & 2.642 .871 & 14,8 & 4.807 .025 & 15,3 & 81,9 \\
\hline México & 473.923 & 2,7 & 885.456 & 2,8 & 86,8 \\
\hline
\end{tabular}

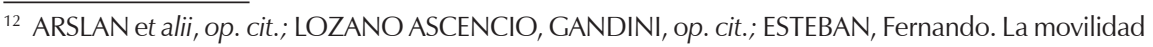
de profesionales y estudiantes universitarios latinoamericanos y caribeños a países de la OCDE. 


\begin{tabular}{|c|c|c|c|c|c|}
\hline Centroamérica & 247.091 & 1,4 & 438.979 & 1,4 & 77,7 \\
\hline Belice & 8.662 & 0 & 13.508 & 0 & 55,9 \\
\hline Costa Rica & 18.472 & 0,1 & 30.286 & 0,1 & 64 \\
\hline El Salvador & 64.340 & 0,4 & 128.780 & 0,4 & 100,2 \\
\hline Guatemala & 40.565 & 0,2 & 79.556 & 0,3 & 96,1 \\
\hline Honduras & 29.146 & 0,2 & 59.755 & 0,2 & 105 \\
\hline Nicaragua & 39.916 & 0,2 & 69.317 & 0,2 & 73,7 \\
\hline Panamá & 45.990 & 0,3 & 57.777 & 0,2 & 25,6 \\
\hline Caribe & 823.326 & 4,6 & 1.341 .232 & 4,3 & 62,9 \\
\hline Antigua y Barbuda & 6.467 & 0 & 8.275 & 0 & 28 \\
\hline Bahamas & 8.867 & 0 & 13.224 & 0 & 49,1 \\
\hline Barbados & 23.243 & 0,1 & 30.145 & 0,1 & 29,7 \\
\hline Cuba & 221.051 & 1,2 & 341.525 & 1,1 & 54,5 \\
\hline Dominica & 5.570 & 0 & 11.781 & 0 & 111,5 \\
\hline Rep. Dominicana & 85.668 & 0,5 & 168.340 & 0,5 & 96,5 \\
\hline Granada & 10.800 & 0,1 & 14.735 & 0 & 36,4 \\
\hline Guyana & 76.019 & 0,4 & 114.273 & 0,4 & 50,3 \\
\hline Haití & 92.564 & 0,5 & 167.283 & 0,5 & 80,7 \\
\hline Jamaica & 190.712 & 1,1 & 292.117 & 0,9 & 53,2 \\
\hline San Cristóbal y Nieves & 5.318 & 0 & 6.419 & 0 & 20,7 \\
\hline Santa Lucía & 4.974 & 0 & 9.309 & 0 & 87,2 \\
\hline Santa Vicente & 8.532 & 0 & 11.727 & 0 & 37,4 \\
\hline Surinam & 2.194 & 0 & 36.502 & 0,1 & 1563,7 \\
\hline Trinidad y Tobago & 81.347 & 0,5 & 115.577 & 0,4 & 42,1 \\
\hline Países andinos & 474.205 & 2,7 & 1.053 .448 & 3,4 & 122,2 \\
\hline Bolivia & 22.579 & 0,1 & 63.137 & 0,2 & 179,6 \\
\hline Colombia & 171.759 & 1 & 374.286 & 1,2 & 117,9 \\
\hline Ecuador & 75.649 & 0,4 & 145.993 & 0,5 & 93 \\
\hline Perú & 118.658 & 0,7 & 260.318 & 0,8 & 119,4 \\
\hline Venezuela & 85.560 & 0,5 & 209.714 & 0,7 & 145,1 \\
\hline Sudamérica & 332.395 & 1,9 & 696.411 & 2,2 & 109,5 \\
\hline Argentina & 105.211 & 0,6 & 247.331 & 0,8 & 135,1 \\
\hline Brasil & 140.764 & 0,8 & 282.180 & 0,9 & 100,5 \\
\hline Chile & 62.072 & 0,3 & 109.046 & 0,3 & 75,7 \\
\hline Paraguay & 4.806 & 0 & 16.295 & 0,1 & 239,1 \\
\hline Uruguay & 19.542 & 0,1 & 41.559 & 0,1 & 112,7 \\
\hline Otros & 291.931 & 1,6 & 391.499 & 1,2 & 34,1 \\
\hline N. E. & 269.086 & 1,5 & 7.129 & 0 & \\
\hline
\end{tabular}

Fuente: Elaboración de los autores en base en OCDE: DIOC 2000/01 (archivo A_1_T) y DIOC 2010/11 (archivo A). 
El crecimiento de la migración calificada también se presentó de manera más acentuada entre las mujeres que en los hombres originarios de América Latina y el Caribe, aunque con marcadas diferencias entre las cinco subregiones. En términos generales, la migración femenina calificada de toda la región creció en $88 \%$, pasando de 1.4 a 2.6 millones, superando al de por sí elevado $75 \%$ registrado por la migración masculina, población que se incrementó de 1.2 a 2.1 millones entre 2000 y 2010 (ver tabla 3). En lo que respecta al comportamiento por subregiones, en el tabla 3 se observa que los países andinos fueron los que presentaron el mayor crecimiento de la migración femenina calificada, pues esta población pasó de poco más de 253 mil mujeres calificadas en el año 2000, a casi 590 mil en 2010. Sudamérica es la segunda región con mayor dinamismo de la migración femenina calificada (119.5\%), seguida por México (95.4\%), que se ubica en la tercera posición. En el otro extremo se ubican Centroamérica y el Caribe, con un crecimiento por debajo del promedio regional. Al respecto, llama la atención el poco aporte de la región caribeña, ya que por mucho tiempo fue considerada el corazón de los flujos migratorios femeninos en Latinoamérica.

\section{Tabla 3 - Población migrante calificada nacida en América Latina y el Caribe, residente en países de la OCDE, por sexo según región de origen: 2000 y 2010}

\begin{tabular}{|c|c|c|c|c|c|c|c|c|c|c|}
\hline & \multicolumn{4}{|c|}{2000} & \multicolumn{4}{|c|}{2010} & \multirow{2}{*}{\multicolumn{2}{|c|}{$\begin{array}{c}\text { Cambio \% entre } \\
2000 \text { y } 2010\end{array}$}} \\
\hline & \multicolumn{2}{|c|}{ Hombres } & \multicolumn{2}{|c|}{ Mujeres } & \multicolumn{2}{|c|}{ Hombres } & \multicolumn{2}{|c|}{ Mujeres } & & \\
\hline & Abs. & $\%$ & Abs. & $\%$ & Abs. & $\%$ & Abs. & $\%$ & Hombres & Mujeres \\
\hline $\begin{array}{c}\text { América Latina } \\
\text { y el Caribe }\end{array}$ & 1.221 .815 & 100,0 & 1.421 .056 & 100,0 & 2.138 .290 & 100,0 & 2.668 .735 & 100,0 & 75,0 & 87,8 \\
\hline México & 239.215 & 19,6 & 234.708 & 16,5 & 426.727 & 20,0 & 458.729 & 17,2 & 78,4 & 95,4 \\
\hline Centroamérica & 118.420 & 9,7 & 128.671 & 9,1 & 199.419 & 9,3 & 239.560 & 9,0 & 68,4 & 86,2 \\
\hline Caribe & 357.431 & 29,3 & 465.895 & 32,8 & 571.929 & 26,7 & 769.303 & 28,8 & 60,0 & 65,1 \\
\hline Países andinos & 220.958 & 18,1 & 253.247 & 17,8 & 463.728 & 21,7 & 589.720 & 22,1 & 109,9 & 132,9 \\
\hline Sudamérica & 156.107 & 12,8 & 176.288 & 12,4 & 309.527 & 14,5 & 386.884 & 14,5 & 98,3 & 119,5 \\
\hline Otros & 129.684 & 10,6 & 162.247 & 11,4 & 166.960 & 7,8 & 224.539 & 8,4 & 28,7 & 38,4 \\
\hline
\end{tabular}

Fuente: Elaboración de los autores en base en OCDE: DIOC 2000/01 (archivo A_1_T) y DIOC 2010/11 (archivo A).

Estos cambios en los lugares de origen de la migración calificada en América Latina y el Caribe también se corresponden con cambios en los países y regiones de destino de los migrantes de la región. De acuerdo con la información procesada, Estados Unidos y Canadá constituyen el principal destino de la migración calificada latinoamericana. Entre 2000 y 2010 la población de migrantes latinoamericanos calificados en esos países aumentó de 2.1 a 3.6 millones de personas. No obstante, en términos relativos estos dos países receptores registraron una notable disminución al pasar de albergar $82 \%$ del stock total en 2000 a 74\% en 2010. En contraste, otros países y regiones del mundo 
se han perfilado como nuevos puntos de llegada y otros se han consolidado como destinos de la migración calificada. Tal es el caso de Europa, donde los migrantes calificados latinoamericanos aumentaron su cuantía, tanto en términos absolutos (de 403 mil a poco más de un millón de personas) como en relativos (de $15.3 \%$ a $21.4 \%$ ), situándose como el segundo destino más importante, ya que continua siendo una región receptora de importantes flujos migratorios de la región. Destaca también el crecimiento absoluto que tuvo el stock de migrantes de la región en Asia, el cual si bien no alcanza las dimensiones numéricas que en Estados Unidos, Canadá y Europa, aumentó de 33 mil a casi 54 mil personas, consolidándose claramente como un nuevo destino (ver tabla 4).

\section{Tabla 4 - Población migrante calificada nacida en América Latina y el Caribe, residente en países de la $\mathrm{OCDE}$, por país o región de origen y destino: 2000-2010}

\begin{tabular}{|c|c|c|c|c|c|c|c|c|c|c|}
\hline \multirow{3}{*}{$\begin{array}{l}\text { Región de } \\
\text { origen }\end{array}$} & \multicolumn{10}{|c|}{ Región de destino } \\
\hline & \multicolumn{5}{|c|}{2000} & \multicolumn{5}{|c|}{2010} \\
\hline & Total & $\begin{array}{l}\text { EU y } \\
\text { Canadá }\end{array}$ & Europa & Asia & Otro & Total & $\begin{array}{l}\text { EU y } \\
\text { Canadá }\end{array}$ & Europa & Asia & Otro \\
\hline $\begin{array}{c}\text { Total } \\
\text { (absolutos) }\end{array}$ & 2.637 .711 & 2.155 .555 & 403.072 & 33.155 & 45.929 & 4.807 .025 & 3.581 .228 & 1.024 .071 & 53.828 & 147.898 \\
\hline \multicolumn{11}{|l|}{$\begin{array}{c}\text { Distribución } \\
\text { porcentual } \\
\text { horizontal }\end{array}$} \\
\hline $\begin{array}{c}\text { América Latina } \\
\text { y el Caribe }\end{array}$ & 100,0 & 81,7 & 15,3 & 1,3 & 1,7 & 100,0 & 74,5 & 21,3 & 1,1 & 3,1 \\
\hline México & 100,0 & 96,1 & 3,7 & 0,1 & 0,1 & 100,0 & 93,8 & 5,6 & 0,2 & 0,4 \\
\hline Centroamérica & 100,0 & 93,0 & 3,4 & 0,0 & 3,6 & 100,0 & 91,4 & 5,7 & 0,1 & 2,8 \\
\hline Caribe & 100,0 & 90,1 & 9,3 & 0,0 & 0,6 & 100,0 & 84,5 & 14,7 & 0,1 & 0,7 \\
\hline Países andinos & 100,0 & 68,3 & 27,7 & 1,5 & 2,5 & 100,0 & 58,8 & 34,0 & 1,0 & 6,2 \\
\hline Sudamérica & 100,0 & 51,5 & 35,9 & 7,6 & 5,1 & 100,0 & 40,9 & 45,5 & 5,6 & 8,0 \\
\hline Otros & 100,0 & 81,4 & 17,5 & 0,1 & 1,0 & 100,0 & 79,5 & 19,8 & 0,0 & 0,7 \\
\hline \multicolumn{11}{|l|}{$\begin{array}{c}\text { Distribución } \\
\text { porcentual } \\
\text { vertical }\end{array}$} \\
\hline $\begin{array}{l}\text { América Latina } \\
\text { y el Caribe }\end{array}$ & 100,0 & 100,0 & 100,0 & 100,0 & 100,0 & 100,0 & 100,0 & 100,0 & 100,0 & 100,0 \\
\hline México & 18,0 & 21,1 & 4,3 & 1,6 & 1,2 & 18,4 & 23,2 & 4,8 & 4,1 & 2,1 \\
\hline Centroamérica & 9,4 & 10,7 & 2,1 & - & 19,5 & 9,1 & 11,2 & 2,4 & 0,7 & 8,3 \\
\hline Caribe & 31,2 & 34,4 & 19,0 & - & 10,3 & 27,9 & 31,7 & 19,3 & 2,3 & 6,1 \\
\hline Países andinos & 18,0 & 15,0 & 32,6 & 21,6 & 26,2 & 21,9 & 17,3 & 34,9 & 19,8 & 44,2 \\
\hline Sudamérica & 12,6 & 7,9 & 29,6 & 76,0 & 36,6 & 14,5 & 8,0 & 31,0 & 73,1 & 37,4 \\
\hline Otros & 10,9 & 10,8 & 12,5 & 0,8 & 6,2 & 8,1 & 8,7 & 7,6 & 0,1 & 1,8 \\
\hline
\end{tabular}

Nota: No se incluye a la población que no especificó el lugar de destino en 2000: 5,160 casos.

Fuente: Elaboración de los autores en base en OCDE: DIOC 2000/01 (archivo A_1_T) y DIOC 2010/11 (archivo A). 
Estos cambios en la geografía de la migración calificada por región de destino adquieren distintos matices y dimensiones según países y subregiones de origen en América Latina y el Caribe. De hecho, a partir de la información contenida en la tabla 4 es posible diferenciar dos grandes flujos de la migración calificada latinoamericana. El primero de ellos es el que se dirige a Estados Unidos y Canadá, donde radica poco más del noventa por ciento de los migrantes calificados de México, Centroamérica y el Caribe entre 2000 y 2010. De acuerdo con dicha información, estos migrantes representan alrededor del $66 \%$ del stock de población calificada nativa de la región viviendo en Estados Unidos y Canadá; es decir, en esos dos países, poco más de seis de cada diez migrantes calificados son mexicanos, centroamericanos o caribeños. Estos últimos, tienen además, una alta participación en el flujo migratorio que se dirige hacia Europa, quienes pasaron de representar $9.3 \%$ a $14.7 \%$ en el periodo señalado, y donde representaban cerca del 19\% del total de migrantes calificados en el viejo continente en esos años.

El segundo gran flujo migratorio es, precisamente, el que se dirige a Europa, el cual está compuesto mayoritariamente por personas originarias de los países andinos y de Sudamérica. En los últimos diez años, los primeros tuvieron un crecimiento significativo al punto de que su peso relativo pasó de $27.7 \%$ a $34 \%$. En el año 2000, representaban el 32.6\% del total de los migrantes calificados latinoamericanos radicados en Europa, cifra que aumentó a $34.9 \%$ en 2010. Los segundos, por su parte, también experimentaron un crecimiento relativo importante, que fue de $35.9 \%$ a $45.5 \%$. Sin embargo, continúan representando cerca del 30\% del total de los migrantes de la región en Europa. Ambos grupos poblacionales, por el contrario, disminuyeron su participación en el stock de migrantes calificados en Estados Unidos y Canadá en alrededor de diez puntos porcentuales entre 2000 y 2010. Dicho en otras palabras, en ese periodo, redujeron su participación en el flujo migratorio que se dirige hacia Norteamérica.

Se observa entonces que la geografía de la migración de recursos humanos calificados se está modificando, tanto en los lugares de origen como en los de destino. Entre los cambios más notables se encuentran, por un lado, el crecimiento de la migración calificada en la región Andina, la cual se dirige principalmente a Europa, así como la continua y sostenida migración de sudamericanos, que también se dirige principalmente al viejo continente, aunque con un ligero repunte hacia países asiáticos. Y por otro, una disminución de la migración calificada hacia Estados Unidos y Canadá, incluso en el caso de México, principal emisor de personal calificado hacia esos países. En estos flujos migratorios tienen una alta participación las mujeres calificadas, proceso del cual todavía hace falta mucho por conocer en Latinoamérica. 
Antes de pasar al análisis de la inserción socioeconómica de los migrantes calificados de América Latina en países de la OCDE, quisiéramos finalizar esta sección con una breve reflexión sobre los cambios entre 2000 y 2010, de lo que representa la población de migrantes calificados radicados en el exterior, frente a la población calificada nativa de cada país de ALyC. Un primer hecho a tomar en cuenta es que entre esos años, la región de América Latina y el Caribe presentó un crecimiento de $56 \%$ de su población calificada nativa, al pasar de 34.5 a 53.8 millones. No obstante este significativo aumento, llama la atención que la población de migrantes calificados radicados en los países de la OCDE creció en un porcentaje significativamente mayor (87\%) entre 2000 y 2010.

La tabla 5 presenta los valores de las tasas de emigración calificada (TEC) por países y subregiones de ALyC. Este indicador refleja el peso que tiene la población de emigrantes calificados residentes en el exterior (en este caso en alguno de los 33 países de la OCDE) respecto de la población calificada del mismo país (ya sea migrante o no migrante). Diversos trabajos han utilizado este indicador para apreciar la importancia que tiene la migración de recursos humanos calificados en los diversos países y analizar los cambios en el tiempo ${ }^{13}$. Para el cálculo de las TEC se utilizaron las estimaciones de Barro y Lee Le $^{14}$ de la población de 2000 y 2010 por nivel de escolaridad, residente en los países de $\mathrm{ALyC}$, con excepción de ocho países del Caribe no incluidos en esta fuente de información. La tabla 5 muestra un incremento de las TEC en toda la región de ALyC al pasar de 6.25 a $7.41 \%$, indicador que en todas las subregiones se incrementó, salvo en el caso del Caribe. A pesar de que las subregiones de Centroamérica y el Caribe presentan las TEC más elevadas de la región, destaca que en las regiones Andina y Sudamérica las TEC tuvieron incrementos significativos entre 2000 y 2010 (salvo en el caso de Colombia), lo que indica que la mano de obra calificada formada en los países de origen no se está incorporando en los mercados de trabajo locales y, por el contrario, está intensificado la emigración absoluta y relativa hacia otros países.

${ }^{13}$ DOQUIER, LOWELL, MARFOUK, op. cit.; LOZANO ASCENCIO, GANDINI, op. cit.; BRÜCKER, Herbert, CAPUANO, Stella, MARFOUK, Abdeslam. Education, gender and international migration: Insights from a panel dataset 1980-2010.

${ }^{14}$ BARRO, Robert, LEE, Jong-Wha. A New Data Set of Educational Attainment in the World, 19502010. 
Tabla 5 - Población nativa calificada, migrantes calificados y tasa de emigración calificada por países y subregiones de América Latina y el Caribe, 2000 y 2010 (población en miles)

\begin{tabular}{|c|c|c|c|c|c|c|}
\hline & \multicolumn{3}{|c|}{2000} & \multicolumn{3}{|c|}{2010} \\
\hline & $\begin{array}{c}\text { Población } \\
\text { nativa } \\
\text { calificada }\end{array}$ & $\begin{array}{l}\text { Migrantes } \\
\text { calificados }\end{array}$ & $\begin{array}{c}\text { Tasa de } \\
\text { emigración } \\
\text { calificada }\end{array}$ & $\begin{array}{c}\text { Población } \\
\text { nativa } \\
\text { calificada }\end{array}$ & $\begin{array}{l}\text { Migrantes } \\
\text { calificados }\end{array}$ & $\begin{array}{c}\text { Tasa de } \\
\text { emigración } \\
\text { calificada }\end{array}$ \\
\hline $\begin{array}{l}\text { América Latina y el } \\
\text { Caribe* }\end{array}$ & $34.488,2$ & $2.298,2$ & 6,25 & $53.751,2$ & $4.303,6$ & 7,41 \\
\hline México & $7.314,9$ & 473,9 & 6,08 & $13.563,7$ & 885,5 & 6,13 \\
\hline Centroamérica & $1.718,0$ & 247,1 & 12,57 & $2.221,9$ & 439,0 & 16,50 \\
\hline Belice & 9,0 & 8,7 & 49,15 & 11,3 & 13,5 & 54,47 \\
\hline Costa Rica & 361,7 & 18,5 & 4,86 & 581,1 & 30,3 & 4,95 \\
\hline El Salvador & 358,1 & 64,3 & 15,23 & 281,2 & 128,8 & 31,41 \\
\hline Guatemala** & 174,8 & 40,6 & 18,83 & 207,5 & 79,6 & 27,71 \\
\hline Honduras & 166,6 & 29,1 & 14,89 & 180,1 & 59,8 & 24,92 \\
\hline Nicaragua & 273,4 & 39,9 & 12,74 & 410,5 & 69,3 & 14,45 \\
\hline Panamá & 374,5 & 46,0 & 10,94 & 550,2 & 57,8 & 9,50 \\
\hline Caribe & $1.681,5$ & 770,6 & 31,43 & $2.811,1$ & $1.229,3$ & 30,43 \\
\hline Barbados & 2,3 & 23,2 & 90,92 & 10,4 & 30,1 & 74,32 \\
\hline Cuba & 703,4 & 221,1 & 23,91 & $1.810,1$ & 341,5 & 15,87 \\
\hline Rep. Dominicana & 674,7 & 85,7 & 11,27 & 549,2 & 168,3 & 23,46 \\
\hline Guyana & 0,5 & 76,0 & 99,33 & 2,2 & 114,3 & 98,08 \\
\hline Haití & 36,8 & 92,6 & 71,53 & 53,6 & 167,3 & 75,74 \\
\hline Jamaica & 214,2 & 190,7 & 47,10 & 283,1 & 292,1 & 50,78 \\
\hline Trinidad y Tobago & 49,5 & 81,3 & 62,17 & 102,4 & 115,6 & 53,01 \\
\hline Países andinos & $10.954,8$ & 474,2 & 4,15 & $15.605,2$ & $1.053,4$ & 6,32 \\
\hline Bolivia & 648,2 & 22,6 & 3,37 & $1.338,2$ & 63,1 & 4,51 \\
\hline Colombia & $2.715,4$ & 171,8 & 5,95 & $7.155,3$ & 374,3 & 4,97 \\
\hline Ecuador & $1.126,0$ & 75,6 & 6,30 & 921,0 & 146,0 & 13,68 \\
\hline Perú & $4.507,1$ & 118,7 & 2,57 & $3.504,8$ & 260,3 & 6,91 \\
\hline Venezuela & $1.958,1$ & 85,6 & 4,19 & $2.685,8$ & 209,7 & 7,24 \\
\hline Sudamérica & $12.819,1$ & 332,4 & 2,53 & $19.549,3$ & 696,4 & 3,44 \\
\hline Argentina & $2.319,1$ & 105,2 & 4,34 & $3.414,1$ & 247,3 & 6,75 \\
\hline Brasil & $7.769,5$ & 140,8 & 1,78 & $13.584,4$ & 282,2 & 2,03 \\
\hline Chile & $2.265,8$ & 62,1 & 2,67 & $1.993,9$ & 109,0 & 5,19 \\
\hline Paraguay & 217,3 & 4,8 & 2,16 & 348,0 & 16,3 & 4,47 \\
\hline Uruguay & 247,4 & 19,5 & 7,32 & 208,8 & 41,6 & 16,60 \\
\hline
\end{tabular}

* / La población de migrantes calificados de ALyC no coincide con la de la Tabla 2, ya que no fue incluida la población de ocho países caribeños.

**/ La población nativa calificada de Guatemala para 2010 fue estimada con base en información de 2005.

Fuente: Elaboración de los autores con base en BARRO, Robert, LEE, Jong-Wha. A New Data Set of Educational Attainment in the World y tabla 2. 


\section{Inserción socioeconómica de los migrantes calificados de América Latina y el Caribe en países de la OCDE}

La inserción laboral es y ha sido considerada como uno de los indicadores que mayormente da cuenta de los procesos de integración socioeconómica de los inmigrantes en las sociedades de llegada, ya que a través de ella no sólo es posible conocer el tipo de ocupación o sector económico en el que se desempeñan o son demandados los migrantes, y sus condiciones laborales, sino también porque perfila y determina en gran medida el desarrollo profesional y trayectorias laborales de los migrantes, además porque tiene efectos directos e indirectos en otros ámbitos de la vida personal y familiar; por ejemplo, en el acceso a la salud, jubilaciones y pensiones, tenencia de la vivienda, etc. Sobre este punto, las estimaciones realizadas sobre la inserción laboral de los migrantes calificados muestran que, en perspectiva comparada, los latinoamericanos presentan altas tasas de participación laboral e incluso por arriba de las ostentadas por otros migrantes. En el año 2000, la tasa de participación económica fue de 75\%, y se elevó a $79 \%$ en 2010 , ambas tasas se encuentran muy por encima del promedio total, lo que ubica a los migrantes calificados de ALyC como el segundo grupo poblacional con la tasa de participación económica más alta en ambos años, sólo por debajo de los nativos de Oceanía. En términos absolutos, estas cifras estarían indicando que en el periodo señalado la región latinoamericana pasó de contribuir con poco más de 197 mil trabajadores en el año 2000 a poco más de 384 mil en 2010, los cuales se sumaron al contingente de mano de obra calificada que se desempeña en los mercados laborales de los países de la OECD. En concordancia con lo anterior, también registraron una de las tasas más bajas de inactividad económica, la cual disminuyó de $25 \%$ a 20.5\% entre 2000 y 2010, mientras que en otros grupos de migrantes dicho indicador se mantuvo (ver tabla 6).

Esta información permite suponer que la población calificada latinoamericana además de ser selectiva en cuanto a sus niveles de capital humano, lo es también en términos de su participación laboral; es decir, en cuanto a su aportación a la fuerza laboral de los países de llegada. Del total de los migrantes latinoamericanos que formaban parte de la población económicamente activa en 2000, alrededor de nueve de cada diez trabajaba o se encontraba ocupado (94.5\%), porcentaje muy similar al presentado por otros migrantes. No obstante, dicha cifra disminuyó a 90.8\% en 2010, posiblemente a consecuencia de la crisis económica mundial de 2008, que como se puede ver en la tabla 6 afectó a todos los migrantes calificados, pero fueron los latinoamericanos y africanos los que se vieron más afectados por este fenómeno coyuntural: en los primeros la tasa de desempleo aumentó de 5.5\% a $9.2 \%$, mientras que en los segundos se elevó de $8.6 \%$ a 10.8\% en el periodo analizado. En el caso específico de los latinoamericanos, dicho indicador se ubica por encima del promedio total en 2010, cuando una década antes la 
situación era a la inversa. Ello apunta a que ambos grupos poblacionales, a diferencia de otros migrantes calificados, son más vulnerables a caer en desempleo y, por ende, en situaciones de precariedad laboral. Por ejemplo, en comparación con los nativos de Asia y Oceanía, entre los cuales la tasa de desempleo se mantuvo casi estable en el periodo, a pesar de la severidad de la crisis económica en todos los países del mundo (ver tabla 6).

\section{Tabla 6 - Población migrante de alta calificación, residente en países de la OECD, por condición de actividad, según región de nacimiento: 2000 y 2010}

\begin{tabular}{|c|c|c|c|c|c|c|c|c|}
\hline & Total & África & Asia & Europa & $\begin{array}{c}\text { E.U. y } \\
\text { Canadá }\end{array}$ & $\begin{array}{l}\text { América } \\
\text { Latina y } \\
\text { el Caribe }\end{array}$ & Oceanía & N.E. \\
\hline \multicolumn{9}{|c|}{2000} \\
\hline Total & 17.860 .010 & 1.652 .823 & 5.933 .383 & 6.108 .029 & 807.276 & 2.635 .326 & 303.592 & 419.581 \\
\hline PEA & 13.220 .073 & 1.309 .862 & 4.356 .655 & 4.452 .264 & 596.640 & 1.976 .324 & 253.734 & 274.594 \\
\hline Ocupados & 12.433 .562 & 1.196 .763 & 4.140 .338 & 4.214 .909 & 577.540 & 1.866 .697 & 243.245 & 194.070 \\
\hline Desocupados & 786.511 & 113.099 & 216.317 & 237.355 & 19.100 & 109.627 & 10.489 & 80.524 \\
\hline PNEA & 4.634 .232 & 342.447 & 1.574 .489 & 1.653 .543 & 210.356 & 658.789 & 49.704 & 144.904 \\
\hline N.E. & 5.705 & 514 & 2.239 & 2.222 & 280 & 213 & 154 & 83 \\
\hline Total & 100,0 & 100,0 & 100,0 & 100,0 & 100,0 & 100,0 & 100,0 & 100,0 \\
\hline PEA & 74,0 & 79,2 & 73,4 & 72,9 & 73,9 & 75,0 & 83,6 & 65,4 \\
\hline Ocupados & 94,1 & 91,4 & 95,0 & 94,7 & 96,8 & 94,5 & 95,9 & 70,7 \\
\hline Desocupados & 5,9 & 8,6 & 5,0 & 5,3 & 3,2 & 5,5 & 4,1 & 29,3 \\
\hline PNEA & 25,9 & 20,7 & 26,5 & 27,1 & 26,1 & 25,0 & 16,4 & 34,5 \\
\hline N.E. & 0,0 & 0,0 & 0,0 & 0,0 & 0,0 & 0,0 & 0,1 & 0,0 \\
\hline \multicolumn{9}{|c|}{2010} \\
\hline Total & 31.767 .368 & 3.143 .366 & 11.080 .351 & 10.828 .800 & 1.156 .761 & 4.842 .969 & 509.594 & 205.527 \\
\hline PEA & 23.963 .262 & 2.466 .896 & 8.231 .983 & 7.985 .674 & 850.898 & 3.847 .445 & 421.934 & 158.432 \\
\hline Ocupados & 22.173 .632 & 2.200 .470 & 7.684 .112 & 7.438 .212 & 810.263 & 3.493 .063 & 402.625 & 144.887 \\
\hline Desocupados & 1.789 .630 & 266.426 & 547.871 & 547.462 & 40.635 & 354.382 & 19.309 & 13.545 \\
\hline PNEA & 7.792 .900 & 675.974 & 2.842 .102 & 2.839 .768 & 305.452 & 995.214 & 87.295 & 47.095 \\
\hline N.E. & 11.206 & 496 & 6.266 & 3.358 & 411 & 310 & 365 & 0 \\
\hline Total & 100,0 & 100,0 & 100,0 & 100,0 & 100,0 & 100,0 & 100,0 & 100,0 \\
\hline PEA & 75,4 & 78,5 & 74,3 & 73,7 & 73,6 & 79,4 & 82,8 & 77,1 \\
\hline Ocupados & 92,5 & 89,2 & 93,3 & 93,1 & 95,2 & 90,8 & 95,4 & 91,5 \\
\hline Desocupados & 7,5 & 10,8 & 6,7 & 6,9 & 4,8 & 9,2 & 4,6 & 8,5 \\
\hline PNEA & 24,5 & 21,5 & 25,6 & 26,2 & 26,4 & 20,5 & 17,1 & 22,9 \\
\hline N.E. & 0,0 & 0,0 & 0,1 & 0,0 & 0,0 & 0,0 & 0,1 & 0,0 \\
\hline
\end{tabular}

Fuente: Elaboración de los autores en base en OCDE: DIOC 2000/01 (archivo G_T) y DIOC 2010/11 (archivo C). 
Al enfocar la mirada en América Latina y el Caribe se observa, de manera general, un incremento de la población económicamente activa y, por el contrario, un descenso de la población migrante calificada inactiva según país y subregión de origen entre 2000 y 2010; es decir, un patrón de participación laboral similar al promedio regional. Sin embargo, resaltan diferencias importantes en cuanto a los niveles de ocupación y desocupación. Los originarios de México son el grupo que tuvo el porcentaje más alto de ocupados y en consecuencia el más bajo de desocupados durante la última década, seguidos muy de cerca por los de Centroamérica, con excepción de los procedentes de Honduras y Nicaragua, entre quienes el desempleo aumentó ligeramente. Los originarios del Caribe también exhiben altos porcentajes de población ocupada, pero se han visto más abatidos por el desempleo que los mexicanos y centroamericanos, el cual afectó principalmente a los migrantes calificados de Republica Dominicana, Cuba y Haití. Estas tendencias son hasta cierto punto sorprendentes ya que como se señaló líneas arriba, el destino principal de la migración calificada de estos países y subregiones es Estados Unidos, nación donde tuvo su epicentro la crisis económica de 2008, la cual impactó negativamente a sectores económicos donde se insertan laboralmente los migrantes latinoamericanos en ese país. Esto hace suponer que los migrantes calificados no se vieron afectados por la crisis de la misma forma que sus connacionales de menor calificación (ver tabla 7).

Una situación muy distinta se presenta en el caso de los migrantes calificados de los países andinos y sudamericanos, quienes radican en mayor medida en países europeos. Estos migrantes se han visto más afectados por el desempleo. Así, por ejemplo, entre los originarios de los países andinos tal situación aumentó de $7.8 \%$ a $12.4 \%$ entre 2000 y 2010 , siendo los nativos de Bolivia, Ecuador y Venezuela los que experimentaron el mayor desempleo durante la década. En el caso de los migrantes calificados provenientes de la región sudamericana, el porcentaje de desempleados se acrecentó de $6.5 \%$ a $11.7 \%$, y dentro de este grupo poblacional, tal problemática laboral envolvió principalmente a los migrantes de Paraguay, Argentina y Uruguay, situándose muy por encima del promedio subregional y del promedio total de la región en 2010 (ver tabla 7).

Tabla 7 - Población migrante de alta calificación, residente en países de la OECD, por condición de actividad, según región y país de nacimiento: 2000 y 2010

\begin{tabular}{l|c|c|c|c|c|c|c|c}
\hline & \multicolumn{3}{|c|}{2000} & \multicolumn{4}{c}{2010} \\
\hline & PEA & PNEA & $\begin{array}{c}\% \text { de } \\
\text { ocupados }\end{array}$ & $\begin{array}{c}\text { \% de } \\
\text { desocupado }\end{array}$ & PEA & PNEA & $\begin{array}{c}\% \text { de } \\
\text { ocupados }\end{array}$ & $\begin{array}{c}\% \text { de } \\
\text { desocupado }\end{array}$ \\
\hline $\begin{array}{l}\text { América Latina y el } \\
\text { Caribe }\end{array}$ & 75,00 & 25,00 & 94,5 & 5,5 & 79,4 & 20,5 & 90,8 & 9,2 \\
\hline México & 71,4 & 28,6 & 94,8 & 5,2 & 78,9 & 21,1 & 93,2 & 6,8 \\
\hline Centroamérica & 76,0 & 24,0 & 95,0 & 5,0 & 80,8 & 19,2 & 92,8 & 7,2 \\
\hline
\end{tabular}




\begin{tabular}{|c|c|c|c|c|c|c|c|c|}
\hline Belice & 82,0 & 18,0 & 94,3 & 5,7 & 79,6 & 20,4 & 94,2 & 5,8 \\
\hline Costa Rica & 74,2 & 25,7 & 96,2 & 3,8 & 76,7 & 23,3 & 93,7 & 6,3 \\
\hline El Salvador & 77,1 & 22,9 & 94,3 & 5,7 & 83,9 & 16,1 & 92,9 & 7,1 \\
\hline Guatemala & 74,3 & 25,7 & 94,6 & 5,4 & 81,9 & 18,0 & 93,3 & 6,7 \\
\hline Honduras & 74,5 & 25,5 & 94,5 & 5,5 & 80,0 & 20,0 & 91,6 & 8,4 \\
\hline Nicaragua & 74,6 & 25,4 & 94,8 & 5,2 & 80,9 & 19,1 & 91,7 & 8,3 \\
\hline Panamá & 77,4 & 22,6 & 96,7 & 3,3 & 75,5 & 24,5 & 93,7 & 6,3 \\
\hline Caribe & 77,2 & 22,8 & 94,9 & 5,1 & 79,0 & 21,0 & 91,7 & 8,3 \\
\hline Antigua y Barbuda & 83,5 & 16,5 & 96,1 & 3,9 & 77,8 & 22,2 & 95,1 & 4,9 \\
\hline Bahamas & 76,5 & 23,5 & 95,4 & 4,6 & 83,3 & 16,7 & 94,2 & 5,8 \\
\hline Barbados & 78,4 & 21,6 & 96,0 & 4,0 & 71,8 & 28,2 & 93,8 & 6,2 \\
\hline Cuba & 69,6 & 30,3 & 94,8 & 5,2 & 74,4 & 25,6 & 89,9 & 10,1 \\
\hline Dominica & 82,5 & 17,5 & 93,6 & 6,4 & 82,6 & 17,4 & 91,6 & 8,4 \\
\hline Rep. Dominicana & 73,6 & 26,4 & 92,8 & 7,2 & 81,9 & 18,1 & 89,6 & 10,4 \\
\hline Granada & 81,8 & 18,2 & 95,3 & 4,7 & 77,0 & 23,0 & 93,7 & 6,3 \\
\hline Guyana & 82,4 & 17,6 & 96,0 & 4,0 & 80,0 & 20,0 & 94,2 & 5,8 \\
\hline Haití & 78,3 & 21,7 & 93,4 & 6,6 & 82,7 & 17,3 & 90,4 & 9,6 \\
\hline Jamaica & 82,5 & 17,5 & 95,7 & 4,3 & 81,4 & 18,6 & 92,9 & 7,1 \\
\hline $\begin{array}{l}\text { San Cristóbal y } \\
\text { Nieves }\end{array}$ & 81,3 & 18,7 & 95,8 & 4,2 & 78,7 & 21,3 & 93,4 & 6,6 \\
\hline Santa Lucía & 78,4 & 21,6 & 94,5 & 5,5 & 78,4 & 21,6 & 91,2 & 8,8 \\
\hline Santa Vicente & 81,5 & 18,5 & 94,5 & 5,5 & 76,4 & 23,6 & 91,7 & 8,3 \\
\hline Surinam & 75,4 & 24,6 & 96,5 & 3,5 & 81,2 & 18,8 & 94,9 & 5,1 \\
\hline Trinidad y Tobago & 81,0 & 19,0 & 95,9 & 4,1 & 77,3 & 22,7 & 93,9 & 6,1 \\
\hline Países andinos & 73,5 & 26,5 & 92,2 & 7,8 & 81,8 & 18,1 & 87,6 & 12,4 \\
\hline Bolivia & 73,5 & 26,4 & 93,7 & 6,3 & 82,7 & 17,3 & 85,6 & 14,4 \\
\hline Colombia & 71,4 & 28,6 & 91,7 & 8,3 & 81,1 & 18,9 & 88,4 & 11,6 \\
\hline Ecuador & 75,0 & 25,0 & 91,9 & 8,1 & 82,2 & 17,8 & 85,9 & 14,1 \\
\hline Perú & 76,1 & 23,8 & 93,2 & 6,8 & 82,8 & 17,2 & 88,6 & 11,4 \\
\hline Venezuela & 72,8 & 27,2 & 91,9 & 8,1 & 81,6 & 18,4 & 86,7 & 13,3 \\
\hline Sudamérica & 74,8 & 25,2 & 93,5 & 6,5 & 78,8 & 21,2 & 88,3 & 11,7 \\
\hline Argentina & 73,5 & 26,5 & 92,4 & 7,6 & 79,7 & 20,3 & 86,7 & 13,3 \\
\hline Brasil & 74,9 & 25,0 & 94,3 & 5,7 & 78,4 & 21,6 & 90,0 & 10,0 \\
\hline Chile & 75,8 & 24,2 & 93,9 & 6,1 & 75,8 & 24,2 & 88,7 & 11,3 \\
\hline Paraguay & 79,7 & 20,3 & 93,7 & 6,3 & 84,3 & 15,7 & 85,3 & 14,7 \\
\hline Uruguay & 77,2 & 22,8 & 93,4 & 6,6 & 82,3 & 17,7 & 87,3 & 12,7 \\
\hline Otros & 76,4 & 23,6 & 96,6 & 3,4 & 75,6 & 24,4 & 93,1 & 6,9 \\
\hline
\end{tabular}

Fuente: Elaboración de los autores en base en OCDE: DIOC 2000/01 (archivo G_T) y DIOC 2010/11 (archivo C). 
Sin embargo, el desempleo no es el único problema que afecta negativamente en la integración económica de los migrantes calificados en los países de la OECD, sino que también se enfrentan a otros problemas como el subempleo y la descualificación, situaciones que ya hemos documentado en otros estudios realizados sobre las condiciones de ocupación y subutilización de habilidades de los migrantes mexicanos calificados en Estados Unidos ${ }^{15}$, las cuales coinciden con los resultados de investigaciones realizadas en otros países y con otros grupos de migrantes con altos niveles de formación educativa ${ }^{16}$. Un renglón en que coinciden estos estudios es aquel en el que se subraya que muchos migrantes calificados realizan trabajos para los cuales se encuentran "sobrecalificados" o bien se encuentran subempleados en actividades económicas cuyo nivel de empleo, en términos de duración o de la productividad, se sitúa por debajo de sus capacidades y preferencias ${ }^{17}$; es decir, se ven perjudicados por el problema de la "sobre-calificación" (overqualification).

Empero, cabe precisar que dicha problemática es vivida por los migrantes calificados de distinta forma y magnitud según país de origen, género y área de formación profesional ${ }^{18}$. Centrando la atención sobre este punto, la información procesada muestra que el problema de la "sobre-calificación" envuelve a una proporción importante de los migrantes calificados residentes en países de la OECD. De tal forma que en 2010, de los 21.9 millones de migrantes calificados que se encontraban empleados, sólo 14 millones trabajaban en ocupaciones propias de su nivel de profesional, y los restantes 7.7 millones lo hacían en ocupaciones para las cuales no se necesitaba de sus capacidades y conocimientos adquiridos durante su formación profesional. Sin embargo, esta problemática es más crítica entre los migrantes calificados provenientes de América Latina y el Caribe, entre los cuales alrededor del $46 \%$ de la población ocupada realizaba una actividad económica para la cual estaban más preparados. Dicho en términos absolutos, poco más de 1.6 millones de

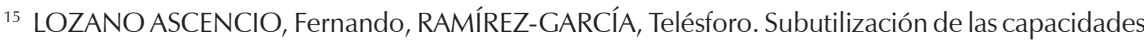
de los profesionales mexicanos de las ciencias y la tecnología y su vínculo con la migración a los Estados; LOZANO ASCENCIO, Fernando, GANDINI, Luciana, RAMÍREZ-GARCíA, Telésforo. Devaluación del trabajo de posgraduados en México y su relación con la migración internacional: el caso de los profesionistas en ciencia y tecnología.

${ }^{16}$ MATTOO, Aaditya, NEAGU, Ileana, ÖZDEN, Çaglar. Brain waste? Educated immigrants in the U.S. Labor Market; BAUDER, Harald. 'Brain abuse', or the devaluation of immigrant labor in Canada; SALAMI, Bukola, NELSON, Sioban. The downward occupational mobility of internationally educated nurses to domestic workers; GREEN, Colin, KLER, Parvinder, LEEVES, Gareth. Immigrant overeducation: evidence from recent arrivals to Australia; FERNÁNDEZ, Cristina, ORTEGA, Carolina. Labor Market Assimilation of Immigrants in Spain: Employment at the Expense of Bad Job-matches?

${ }^{17}$ Organización Internacional del Trabajo (OIT). Informe I: La medición del subempleo. Decimosexta Conferencia Internacional de Estadísticas del Trabajo.

18 RAMÍREZ-GARCÍA, Telésforo, GANDINI, Luciana. Trabajadoras calificadas: las mujeres mexicanas en el mercado de trabajo estadounidense en perspectiva comparada. 
migrantes calificados de la región se encontraban en esa situación laboral. En contraste, los nativos de Estados Unidos y Canadá, así como los procedentes de Oceanía, son los que presentan el menor porcentaje de migrantes calificados que se emplean en trabajos para los cuales se encuentran "sobre-calificados" (20.4\% y $27.4 \%$, respectivamente). En ambos casos, dicha cifra es mucho menor que el promedio total $(35.1 \%)$ y también es, por mucho, menor a la registrada entre migrantes de América Latina y el Caribe, África, Asia y Europa (ver tabla 8). De la lectura de estos datos podría inferirse que el deterioro de la calidad del empleo de los migrantes calificados ha sido más acentuado entre los latinoamericanos.

\section{Tabla 8 - Población migrante de alta calificación, residente en países de la OECD, por nivel de calificación de la ocupación, según país y región de nacimiento, 2010}

\begin{tabular}{|c|c|c|c|c|c|c|c|c|}
\hline & Total & África & Asia & Europa & $\begin{array}{l}\text { E.U. y } \\
\text { Canadá }\end{array}$ & $\begin{array}{l}\text { América } \\
\text { Latina y } \\
\text { el Caribe }\end{array}$ & Oceanía & N.E. \\
\hline Total & 21.955 .692 & 2.114 .509 & 7.633 .695 & 7.474 .053 & 817.445 & 3.487 .437 & 420.970 & 7.583 \\
\hline $\begin{array}{l}\text { No sobre } \\
\text { calificado }\end{array}$ & 14.065 .486 & 1.384 .236 & 4.918 .655 & 4.964 .738 & 642.415 & 1.851 .487 & 299.079 & 4.876 \\
\hline $\begin{array}{l}\text { Sobre- } \\
\text { calificado }\end{array}$ & 7.712 .532 & 718.712 & 2.662 .697 & 2.434 .342 & 166.615 & 1.612 .696 & 115.191 & 2.279 \\
\hline N.E. & 177.674 & 11.561 & 52.343 & 74.973 & 8.415 & 23.254 & 6.700 & 428 \\
\hline Total & 100,0 & 100,0 & 100,0 & 100,0 & 100,0 & 100,0 & 100,0 & 100,0 \\
\hline $\begin{array}{l}\text { No sobre } \\
\text { calificado }\end{array}$ & 64,1 & 65,5 & 64,4 & 66,4 & 78,6 & 53,1 & 71,0 & 64,3 \\
\hline $\begin{array}{l}\text { Sobre- } \\
\text { calificado }\end{array}$ & 35,1 & 34,0 & 34,9 & 32,6 & 20,4 & 46,2 & 27,4 & 30,1 \\
\hline N.E. & 0,8 & 0,5 & 0,7 & 1,0 & 1,0 & 0,7 & 1,6 & 5,6 \\
\hline
\end{tabular}

Fuente: Elaboración de los autores en base en OCDE: DIOC 2010/11 (archivo D).

\section{Comentario final}

Las transformaciones en el patrón migratorio entre 2000 y 2010, tanto en el orden global como específicamente en la región de América Latina y el Caribe, hablan de la existencia de un nuevo escenario migratorio, caracterizado por el importante crecimiento de la migración altamente calificada (grupo conformado por personas con educación terciaria), a un ritmo incluso más acelerado que la migración de media y baja calificación. La evidencia aquí mostrada también da cuenta de un aumento en la tasa de emigración calificada (TEC) entre 2000 y 2010 en prácticamente todos los países de ALyC, lo que significa que una proporción cada vez mayor de profesionistas emigra al extranjero en vez de permanecer en sus países de origen. Por sí misma, la salida de recursos 
humanos altamente calificados es un problema serio en la región, sin embargo, el problema más grave son las condiciones desfavorables en que las personas migrantes se incorporan en el mercado laboral de los países empleadores. En este trabajo se mostró que casi la mitad de los migrantes calificados de ALyC laboran en ocupaciones en las que subutilizan sus conocimientos o habilidades, es decir están sobre-calificados para los trabajos que desempeñan. Este es el porcentaje más alto entre todos los grupos de inmigrantes calificados que laboran en los países de la OCDE.

No obstante la clara tendencia al incremento de la migración calificada hacia los países del Norte Global, es importante señalar que 3 de cada 4 migrantes calificados del mundo, están concentrados en Estados Unidos, país que en la coyuntura actual vive una profunda crisis de su sistema y política migratorios. El ascenso de Donald Trump como presidente de ese país puede implicar cambios en la dinámica y tendencias de la migración en general y de la migración calificada en particular. Una de sus promesas de campaña fue reducir las visas de trabajo para personas de alta calificación (H1B), para empleados de empresas multinacionales (L1), incluso para inversionistas extranjeros (E2), todo ello con el supuesto objetivo de ampliar la oferta de empleos para trabajadores estadounidenses. Más allá de si estas promesas se logran concretar, se trata de un escenario nada favorable para los trabajadores inmigrantes calificados, que podría deteriorar sus condiciones laborales y en general sus condiciones de vida.

\section{Bibliografía}

AMBROSINI, J. William; PERI, Giovanni. The Determinants and the Selection of Mexico-US Migrants. The World Economy, v. 35, n. 2, 2012, p. 111-151.

ANDERSON, Bridget. Migration, immigration controls and the fashioning of precarious workers. Work, employment and society, v. 24, n. 2, 2010, p. 300-317. ANTECOL, Heather; COBB-CLARK, Deborah; TREJO, Stephen. Immigration policy and the skills of immigrants to Australia, Canada and the United States. Journal of Human Resources, v. 38, n. 1, 2003, p. 192-218.

ARSLAN, Cansin; DUMONT, Jean-Christophe; KONE, Zovanga; ÖZDEN, Çağlar; PARSONS, Christopher; XENOGIANI, Theodora. International migration to the OECD in the twenty-first century. Documento de discusión 16. Disponible en: <http://www.knomad.org/docs/skilled_labor_migration/KNOMAD\%20WP\%20 16\%20Migration\%20to\%20OECD_final.pdf>. Acceso en: 10.11.2016.

BARRO, Robert; LEE, Jong-Wha. A New Data Set of Educational Attainment in the World, 1950-2010. Journal of Development Economics, v. 104, 2013, p. 184198. Base de datos actualizada a febrero de 2016.

BERMÚDEZ, Rosa. Trayectorias laborales de migrantes calificadas por razones de estudio. Estudios Demográficos y Urbanos 86, v. 29, n. 2, mayo-agosto 2014, p. 257-299. Disponible en: < http://cedua.colmex.mx/EDU/86/bermudez.pdf>. Acceso en: 12.11.2016. 
BRÜCKER, Herbert; CAPUANO, Stella; MARFOUK, Abdeslam. Education, gender and international migration: Insights from a panel dataset 1980-2010. Methodology Report. Institute for Employment Research, Nürnberg, Alemania, 2013.

CHIQUIAR, Daniel; HANSON, Gordon. International Migration, Self-Selection, and the Distribution of Wages: Evidence from Mexico and the United States. Journal of Political Economy, v. 113, n. 2, 2005, p. 239-281.

COSTA, Daniel; ROSENBAUM, Jennifer. Temporary foreign workers by the numbers. New estimates by visa classification. Washington, D.C.: Economic Policy Institute, 2017.

DOCQUIER, Frédéric; LOWELL, B. Lindsay; MARFOUK, Abdeslam. A Gendered Assessment of Highly Skilled Emigration. Population and Development Review, v. 35, n. 2, Junio 2009, p. 221-447.

ESTEBAN, Fernando. La movilidad de profesionales y estudiantes universitarios latinoamericanos y caribeños a países de la OCDE. Papeles de Población, v. 17, n. 69, julio-diciembre de 2011, p. 105-149. Disponible en: <http://www.scielo.org.mx/scielo. php?script=sci_arttext\&pid=S1405-74252011000300005 > . Acceso en: 12.11.2016.

FERNÁNDEZ, Cristina; ORTEGA, Carolina. Labor Market Assimilation of Immigrants in Spain: Employment at the Expense of Bad Job-matches? Spanish Economic Review, v. 10, n. 2, 2008, p. 83-107. Disponible en: http://link.springer.com/ article/10.1007/s10108-007-9032-4>. Acceso en: 23.01.2017.

GREEN, Colin; KLER, Parvinder; LEEVES, Gareth. Immigrant overeducation: evidence from recent arrivals to Australia. Economics of Education Review, v. 26, n. 4, Agosto 2007, p. 420-432. Disponible en: <http://eprints.lancs. ac.uk/31307/1/Overed_Immigrant_WP.pdf>. Acceso en: 23.01.2017.

KANVUR, Ravin; RAPOPORT, Hillel. Migration selectivity and the evolution of spatial inequality. Journal of Economic Geography, v. 5, n. 1, Enero 2015, p. 43-57.

LOZANO ASCENCIO, Fernando; RAMÍREZ-GARCÍA, Telésforo. Subutilización de las capacidades de los profesionales mexicanos de las ciencias y la tecnología y su vínculo con la migración a los Estados. Notas de Población, año XLII, n. 101, julio-diciembre 2015, p. 157-186. Disponible en: <http://repositorio.cepal.org/ handle/11362/39378>. Acceso en: 7.12.2016.

LOZANO ASCENCIO, Fernando; GANDINI, Luciana; RAMÍREZ-GARCÍA, Telésforo. Devaluación del trabajo de posgraduados en México y su relación con la migración internacional: el caso de los profesionistas en ciencia y tecnología. Migración y Desarrollo, v. 13, n. 25, 2015, p. 61-89. Disponible en: <http://www.scielo.org. $\mathrm{mx} /$ scielo.php?script $=$ sci_arttext\&pid=S187075992015000200061 >. Acceso en: 01.01.2017.

LOZANO ASCENCIO, Fernando; GANDINI, Luciana. Migrantes calificados de América Latina y el Caribe ¿Capacidades desaprovechadas? México: Centro Regional de Investigaciones Multidisciplinarias de la UNAM, 2010.

MATTOO, Aaditya; NEAGU, lleana; ÖZDEN, Çaglar. Brain waste? Educated immigrants in the U.S. Labor Market. Journal of Development Economics, v. 87. Disponible en: $<$ http://dx.doi.org/10.1596/1813-9450-3581>. Acceso en: 07.12.2016. 
MCKENZIE, David; RAPOPORT, Hillel. Self-selection patterns in Mexico-U.S. migration: the role of networks. The Review of Economics and Statistics, v. 4, November 2010, p. 811-821. Disponible en: <http://www.biu.ac.il/soc/ec/students/ teach/835/ data/2012/RESTAT\%202010\%20Networks.pdf>. Acceso en: 21.11.2016.

Organización Internacional del Trabajo (OIT). Informe I: La medición del subempleo. Decimosexta Conferencia Internacional de Estadísticas del Trabajo. Ginebra. Disponible en: <http://www.ilo.org/wcmsp5/groups/public/---dgreports/---stat/ documents/meetingdocument/wcms_088365.pdf>. Acceso en: 01.12.2016.

Organización Internacional para las Migraciones (OIM). Las migraciones en América Latina y el Caribe. Documento de discusión. Disponible en: < http://www.cepal. org/ mujer/reuniones/mesa38/oim_migraciones.pdf> . Acceso en: 18.11.2016.

Organización para la Cooperación y el Desarrollo Económicos (OCDE). Database on Immigrants in OECD countries (DIOC). Disponible en: <http://www.oecd. org/els/ mig/dioc.htm>. Acceso en: marzo de 2016.

ORRENIUS, Pia; ZAVODNY, Madeline. Self-selection among undocumented immigrants from Mexico. Journal of Development Economics, n. 78, 2005, p. 215240. Disponible en: <http://ecademy.agnesscott.edu/ mzavodny/documents/ jde_selfselectionamongun docimmigs_001.pdf>. Acceso en: 29.10.2016.

RAMÍREZ-GARCÍA, Telésforo; GANDINI, Luciana. Trabajadoras calificadas: las mujeres mexicanas en el mercado de trabajo estadounidense en perspectiva comparada. Revista Latinoamericana de Población, v. 10, n. 19, julio-diciembre 2016, p. 33-56. Disponible en: <http://revistarelap.org/ojs/index.php/relap/ article/view/152/154>. Acceso en: 23.01.2017.

RENDALL, Michael S.; PARKER, Susan. Two Decades of Negative Educational Selectivity of Mexican Migrants to the United States. Population and Development Review, v. 40, n. 3, Septiembre 2014, p. 421-446.

RODGERS, Gerry; RODGERS, Janine. Precarious Jobs in Labour Market Regulation: The Growth of Atypical Employment in Western Europe. Brussels: International Labour Organisation, 1989.

Recibido para publicación en 31.01.2017

Aceptado para publicación en 15.03.2017 Received for publication in January 315, 2017 Accepted for publication in March 15th, 2017

ISSN impresso 1980-8585

ISSN eletrônico 2237-9843

http://dx.doi.org/10.1590/1980-85852503880004907 